\title{
Progress of antiviral mechanisms in the mulberry silkworm: A review
}

\author{
Feng Fan, Hu Ping and Chen Keping*
}

School of Food and Biological Engineering, Jiangsu University, 301\# Xuefu Road, Zhenjiang 212013, Jiangsu Province, PR China.

Accepted 21 February, 2013

\begin{abstract}
The silkworm Bombyx mori is both an important economic insect and also exploited as a powerful biological model system. Silkworm larvae often suffer from viral infections, especially the nuclear polyhedrosis virus, causing great losses to the sericulture. Insects have evolved many different ways to defend themselves against pathogens like fungi, bacteria, nematodes, but little is known about the insect immune response against viral infections. To date, several efforts have been made by very few workers to identify the proteins related to the resistance of baculovirus in the silkworm, but no satisfactory researches are reported about the resistant gene of baculovirus. In the present article, the ways of viral infection and certain antiviral proteins involved in silkworm immunity are discussed.
\end{abstract}

Key words: Silkworm, baculovirus, antiviral activity.

\section{INTRODUCTION}

The silkworm Bombyx mori has been exploited as a silk producer for over 5000 years. It is an economically important insect and a model insect for studies in entomology, molecular biology and biotechnology (Tamura et al., 2000; Xiang, 1995). The Bombyx mori nucleopolyhedrovirus (BmNPV) is a principal pathogen of the domestic silkworm. BmNPV often cause severe losses in sericulture, but there are currently no therapeutic agents that can effectively control BmNPV infection. The mechanism by which the silkworm resist viral infections and recruits immune cells to the infective foci or clears infected cells is poorly understood (Popham et al., 2004). Through the investigation of literature related with the silkworm viral resistance, only a few strains of insects show highly resistance to BmNPV infection (Chen et al., 1996; Sunagar et al., 2011), and this is very important basis for further research of silkworm viral resistance.

Insects seemingly lack any adaptive immune responses that operate analogously to the well documented antibody or histocompatibility adaptive immune responses as in vertebrates (Hoffmann, 2003). But it has evolved many different ways to defend themselves against pathogens like fungi, bacteria, nematodes and viruses. Many insects show developmental resistance to baculovirus infection with decreasing susceptibilities at older larval stages (Kirkpatrick et al., 1998; Teakle et al., 1986). The immunity plays an important role in the interaction between the host and pathogen as a part of survival strategy including physical blockades such as peritrophic matrix, epithelial barriers, protease cascades leading to coagulation and melanization, cellular responses such as phagocytosis and encapsulation and also the production of certain antimicrobial peptides (Lavine and Strand, 2002; Lehane, 1997; Levashina et al., 2001; Ligoxygakis et al., 2002; Meister and Lagueux, 2003; Moreno-Habel et al., 2012; Tzou et al., 2000; Vernick et al., 1995), but reports on antiviral mechanisms are little. It is truly surprising to note that the baculovirus protein expression systems are world widely used in research and produc- 
tion, there are virtually little research dealing with the mechanisms of antiviral responses except the resistant gene $n s d-2$ cloned in silkworm by lto et al. (2008). This means that special attention must be focused on this area to understand which genes or proteins of insects involved in resistance control of virus. Apart from this, insects have their potential use as therapeutic agents in clinical applications (Choudhari et al., 2012; Kim et al., 2011). Several antiviral compounds purified from insects have been identified as a small molecular polypeptide (Bulet et al., 1999; Chernysh et al., 2002; Hara and Yamakawa, 1995a, b), and this is an important discovery of immense medical importance (Ourth, 2004).

Baculovirus were considered to be robust against the development of insect resistance (Moscardi, 1999), and several insects were reported to emerge the ability of baculovirus resistance (Fuxa and Richter, 1998; Kenneth et al., 2001; Trudeau et al., 2001), so proper understanding of host-pathogen interaction and anti-defensive molecules released by pathogen to suppress host immunity is necessary before stepping into other aspects like disease control. In this paper, recent progress in antiviral and antimicrobial proteins in insects is discussed. On this direction, we initiated studies on the antiviral mechanisms against BmNPV in silkworm, and this research gives us a great deal of information about the mechanism of resistance in addition to the inheritance of resistance from our lab (Liu et al., 2010). We hope that knowledge of the silkworm's immune response to virus not only helps in the control of viral diseases in the economically important insect like silkworm, but also helps to use silkworm as a bioreactor for the development of antiviral agents important in human health and welfare.

\section{MODE OF INFECTION}

The baculoviruses are a family of large rod-shaped viruses that contain circular double-stranded genome ranging from $80-180 \mathrm{kbp}$. They have very narrow host range, although AcMNPV infects nearly 30 lepidopteron species. The host range of BmNPV is restricted to $B$. mori larvae (Rahman and Gopinathan, 2004). The virus life cycle involves two distinct forms of virus: occlusion derived virus (ODV) and the budded virus (BV), both forms have different role during pathogenesis (Katsuma et al., 2007; Keddie et al., 1989). The virus particles are embedded in protein matrix which is dissolved in the alkaline environment of the host mid-gut. The primary infection begins when the envelops of occlusion derived virus (ODV) fuse to the columnar epithelial cell membrane of the host intestine, then the ODV are taken into the cell in endosomes. Viral transcription and replication occur in the cell nucleus and new BV particles are budded out from the baso lateral side to spread the infection systemically through glycoprotein GP64, which is important for the infection of neighboring host cells and tissues (Monsma et al., 1996). Cells of the insect tracheal system are the important targets of BV, and their infection is critical for the rapid spread of the virus because they provide access to larval tissues surrounded by basal laminar barriers (Washburn et al., 1995). By the end of the infection cycle, most of the insect's tissues are infected and new Obs (BV and ODV) are produced, which spread into the surrounding environment for its next infection.

\section{PROTEINS RELATED WITH BACULOVIRUS RESISTANCE IN THE NON-CELLULAR STRUCTURE OF SILKWORM AND OTHER INSECTS}

As the primary route of infection is through the diet, there must be some antiviral substances existing in the gut juice of the viral resistant insects. Such antiviral substances are gradually identified and isolated from the haemolymph and gut juice in the silkworm, especially the antiviral substances observed by some earlier workers (Aikawa, 1962).

Hayashiya and Nishida (1976) and Hayama (1978) reported the red fluorescent proteins could inactivate the BmNPV, and they firstly demonstrated the RFPs' biosynthesis required the reaction between chlorophyllide-a and an unknown silkworm protein in vitro. In the synthesis process of this protein, chlorophyll-a of mulberry leaves is first converted into chlorollide-a under the action of light and its further synthesized in the midgut cells, and then released into midgut to form RFPs, which have two absorbance peaks at 280 and $605 \mathrm{~nm}$ wave length. Several works have focused on the study of RFPs physiological activities, which revealed it had antiviral effect against BmNPV, but the exact mechanism is still unknown. The first report about the midgut protein to form an RFP is the epithelial cell membrane 252-kDa (P252), which bind with chlorophyllide to form red fluorescent protein complex with absorbance and fluorescence emission peaks at 600 and $620 \mathrm{~nm}$, respectively. It has significant antimicrobial activity which strongly bind with Cry $1 \mathrm{Aa}$, Cry $1 \mathrm{Ab}$, and Cry1Ac toxins of Bacillus thuringiensis (Pandian et al., 2008). But Sunagar et al. $(2008,2011)$ reported there were multiple forms of red fluorescent proteins (RFPs) observed in the gut juice of the silkworm. Comparing the gut juice of multivoltine with bivoltine silkworm races through the method of electrophoretograms and chromatographic eluate, several fluorescent protein bands were observed and in which only three bands existed in the resistant strain. It can be used as a biomarker to measure the degree of susceptibility of silkworm races to NPV and indicated the complex of different RFP participate in the viral resistant control. However, these RFPs have not been identified, so further efforts are needed to obtain the knowledge about the exact mechanism of this protein inhibiting the multiplication of BmNPV, identifying the genes responsible for production of RFP.

Thereafter, except for the molecular identification and characterization of this protein, several proteins related with the baculovirus resistance have been found in the 
gut juice of silkworm such as lipases, NADPH oxidoreductase and serine proteases (Nakazawa et al., 2004; Ponnuvel et al., 2003; Selot et al., 2007). Lipases perform essential roles in the digestion, transport and processing of dietary lipids in most organisms. They are likely to contribute immune defenses, conceivably acting directly against invading microorganisms. Bmlipase-1, a lipase purified from the digestive juice of $B$. mori larvae proved to have a strong antiviral activity against BmNPV as a physiological barrier at the initial site of viral infection. It showed 56\% homology with Drosophila melanogaster lipase and $21 \%$ homology with human lipase. Northern blot analysis showed that the Bmlipase-1 gene only expressed in the midgut and it has been confirmed to be hormonally regulated. In Drosophila melanagaster, there are four lipase genes found to be induced upon immune challenge (De Gregorio et al., 2002). Apart form this, many lipases have been purified and identified from other organisms, which showed different enzymatic specificities and catalytic rates as a digestive enzyme. Interestingly, lipase-like proteins have been identified from viruses such as Marek's Disease virus and Heliothis virescens (Kamil et al., 2005; Smede et al., 2009), which were essential for virus replication and cell cleavage after infection. They were critical for the formation of extracellular enveloped virion (EEV) from intracellular mature virion (IMV), which were reported to play a role in viral membrane biogenesis and membrane fusion (Blasco and Moss, 1991). These results may indicate the lipase gene has a dual role of virus intrusion and virus resistance in the evolution process between pathogen and host. The NADPH oxidoreductase related with energy metabolism utilizes NADP as cofactor to catalyze the transfer of electrons from one molecule to another oxidant. In silkworm, Ruchita Selot reported a purified protein of $26.5 \mathrm{kDa}$ over expressed in the gut juice of disease-resistant multivoltine races has anti-BmNPV activity, and characterized this protein as a soluble $\mathrm{NADH}$-oxidoreductase-like protein (BmNOX). When the BmN cell infected with pre-treated BmNPV (BmNPV particles incubated with BmNOX), the $\mathrm{NADPH}$ oxidoreductase inhibited the capability of the viral particles to infect $\mathrm{BmN}$ cells in vitro.

Serine proteases are among the group of proteins that participate in regulating various physiological functions including antimicrobial peptide synthesis, haemolymph coagulation and melanization of pathogen surfaces. In the digestive juice of silkworm larvae, Nakazawa et al. (2004) proved the presence of serine protease has strong antiviral activity against BmNPV. They designated it as BmSP-2 showed 94\% homology with B.mori serine protease, and reported there may be about five serine protease isoforms. In most insect species, a variety of serine protease inhibitors (SPIs) have been found to inactivate the serine proteases when they are no longer in need. Zhao et al. (2012) predicted and characterized potential SPI genes based on the genome sequences of silkworm. Microarray and qRT-PCR experiments revealed obvious up-regulation and down-regulation of several SPI genes after infection with Escherichia coli, Bacillus bombysepticus, Beauveria bassiana or B.mori nuclear polyhedrosis virus (BmNPV), which suggested that these SPI genes may be involved in resistance to pathogenic microorganisms.

The first step of pathogen invasion is passing through the peritrophic matrix (PM). The PM is a semi-permeable, non-celluar structure which surrounds the food bolus in insect's midgut, which has pore sizes ranging from 21 to $36 \mathrm{~nm}$ in diameter. It serves several functions, including improvement of digestion, protection against mechanical and chemical damage and serving as a barrier to infection by pathogens (Lehane, 1997). Several researches have focused their attention on the protein composition in the PM and the chitinase $A$ has been proved to disrupt the PM and promote the final liquefaction of infected host larvae. In silkworm, through the shotgun liquid chromatography tandem mass spectrometry approach, $\mathrm{Hu}$ et al. (2012) identified 47 proteins in which $51.1 \%$ had the isoelectric point within the range of 5-7, and most of them were found to be closely related to larval nutrients metabolism and innate immunity. To better understand the role of larval PM in larval physiological activities, there must be further study about the protein interaction between pathogens and their host.

\section{PROTEINS INVOLVED IN BACULOVIRUS RESISTANCE IN THE TISSUES OF SILKWORM AND OTHER INSECTS}

The midgut is an important barrier that has to be passed by a baculovirus to initiate infection. Researches about how does the baculovirus infect the tissue have proved that the columnar cells could be infected with ODVs, by the fusion of its lipid bilayer envelopes with midgut cell membranes (Haas-Stapleton et al., 2003). Resistance of Bombyx mori to B.mori densovirus type 2 (BmDNV-2) is caused by a $6-\mathrm{kb}$ deletion in a gene that encodes a transmembrane protein, which is a functional receptor for the virus in the midgut (Ito et al., 2008). But to the resistance of baculovirus in silkworm, it is still unknown whether the resistance mechanism is caused by the receptor deletion of cell membrane. It was reported that eight genes, gloverin 3 and 4 , lebocin, serpin-5, arylphorin, promoting protein, cathepsin $B$ and actin $A 3$ were significantly up-regulated in the midgut of BmNPV-resistant silkworm strain after infection (Bao et al., 2009), but it is not clear whether the proteins encoded by these genes can function in silkworm resistance against BmNPV infection. Recently, in our laboratory we identified the proteins, caspase- 1 and serine protease, are related with BmNPV resistance through the proteomic method (Qin et al., 2012). These proteins only expressed exclusively in BmNPV-resistant silkworms, but not in BmNPV-susceptible silkworms. There may be different mechanisms of mid gut based virus resistance. Resistance of Agrotis segetum 
larvae to oral infection by Spodoptera exigua multiple nucleopolyhedrovirus (SeMNPV) is midgut based. In bypassing the midgut by intrahemocoelic injection of the budded virus, the second viral phenotype, SeMNPV is able to infect $A$. segetum (Jakubowska et al., 2009). This could provide a good perspective for further study how does the midgut defend the virus attack.

Hemolymph is the key to the virus systemic infection. Insects possess an innate immune system, which is characterized by more or less nonspecific cellular and humoral immune reactions (Nakazawa et al., 2004). Phenoloxidase in the insect's plasma can affect baculovirus infection (Popham et al., 2004), and it has been proved to exhibit virucidal activity against BVs of Helicoverpa zea single nucleopolyhedrosis virus. The positive role of haemocytes in clearing microorganisms from the haemolymph of insects by forming melanotic capsules is very clear (Ashida and Brey, 1997), but the haemocyte role seems to be different among different lepidopteron species. Some studies on baculovirus ecology and physiology explain the possibility of antiviral defense mechanism by hemolin in Lepidoptera. Hemolin composed of four immunoglobulin domains is the only insect member of the immunoglobulin super family reported to be upregulated during an immune response. It binds to bacterial lipopolysaccharides (LPS), lipid A, and hemocytes (Bettencourt et al., 1999). Then hemolin is thought to function as an opsonin or as a pattern recognition molecule and thus to be involved in antiviral immune response (Hirai et al., 2004). To our knowledge there is no single report showing the antiviral activity of hemolin in silkworm, but there must be some other substance participated in the baculovirus resistance. In the codling moth the spread of CpGV infection in susceptible and resistant codling moth was observed by Asser-Kaiser et al. (2011) using a recombinant virus, CpGV-bacmid (bacCpGV ${ }^{\text {hsp- }}$ EGFP), expressing enhanced green fluorescence protein. This result provided strong evidence that the baculovirus could enter into the tissue cell of resistant strain Cydia pomonella $\mathrm{L}$. and the baculovirus resistance was caused by early block of virus replication. Previous researches in our laboratory have traced the spread of virus replication in midgut, hemolymph, and fat body of resistant silkworm strain using quantitative PCR. And several proteins identified in hemolymph are related with the baculovirus resistance in silkworm (unpublished).

Lepidopteron larve resist baculovirus infection by selective apoptosis of infected cells from the midgut epithelial cells and by sloughing off infected cells from the midgut cells. To overcome this defense strategy, baculoviruses have evolved inhibitors of apoptosis, which block cell death. Infection of $S$. frugiperda cells with a mutant of AcMNPV lacking a functional antiapoptotic gene p35 leads to apoptosis and inhibition of OB formation (Clem et al., 1991). But even the virions can successfully enter the host body, how does the host defense mechanism operate to control the multiplication of virions and what are the substances involved in the antiviral mechanism? PKR, an enzyme activated by double-stranded RNA (dsRNA), has been proved to play various important roles in the regulation of translation, transcription, signal transduction and protection against viral infections. It could induce cellular apoptosis to prevent further viral spread in many vertebrates. The cDNA isolated and clone from silkworm showed high similarity in its kinase domain to the vertebrate anti-viral kinase (PKR), but the role of this protein in the silkworm still remains to be established (Prudhomme and Couble, 2002). The baculovirus has developed counter measures to combat the anti-viral defense mechanism of the host by synthesizing antiapoptotic proteins like p35 and inhibitors of apoptosis (IAP), so as to prevent cell death induced by the insect cell apoptotic mechanism (Clem et al., 1991). From an evolutionary point of view, the game between virus invasion and host's counter-invasion should be studied in this area. To date, several studies were made on domesticcated insect $B$. mori, but the mechanism of baculovirus resistance is little known. There are about two points of view that could explain the emergence of baculovirus resistance. One is the midgut receptor deletion and the other is some substance in the silkworm cell that could inhibit the virus replication. Efforts in these two directions should be made to clarify the detail mechanism of baculovirus resistance, which will not only improve the silk industry economy but also human health and welfare.

\section{CONCLUSION}

The silkworm, an important economic insect and lepidopteron model insect, is particularly susceptible to virus diseases, especially due to $B$. mori nucleopolyhedrovirus (BmNPV), which results in great loss in sericulture. Information on the immune system in the silkworm in general and the mechanism of baculovirus resistance is little known. There are several recent reports on the presence of antiviral proteins related with baculovirus resistance, but no direct evidence can prove the resistant mechanism. A number of mechanisms operate at different levels, from the origin infection site of midgut to the perpheral tissues of hemolymph where virus proliferates, propagation and multiplication of the virus. There are a few reports about whether the virus can enter into the cell in baculovirus-resistant insects and how does the host inhibit the virus replication, and this required detailed investigations before stepping into disease control and utilization of silkworm as a bioreactor to produce the antiviral proteins of interest. From the above we discussed the point about the inhibition of virus replication in host was increasingly accepted. In codling moth and Hyphantria cunea, the virus replication has been proved, but the mechanism is still unknown. Several researches including our lab's study have focused their attention on the polymerase of baculovirus to clarify its inhibitor in host such as BmNPV- 
resistant silkworm, and we believed that in the near future the complex mechanism could be clearly proved.

\section{ACKNOWLEDGMENTS}

This work was supported by the National Basic Research Program (973) of China N0. 2012CB114604) and the National Natural Science Foundation of China (NO 31272507).

\section{REFERENCES}

Aikawa M (1962). Antiviral substance in the gut-juice of the silkworm. J. Insect Pathol. 4: 72-76.

Ashida M, Brey PT (1997). Molecular Mechanisms of Immune Responses in Insects. Chapman \& Hall, London, pp. 135-172.

Asser-Kaiser S, Radtke P, El-Salamouny S, Winstanley D, Jehle JA (2011). Baculovirus resistance in codling moth (Cydia pomonella L.) caused by early block of virus replication. Virology 410 (2): 360-367.

Bao YY, Tang XD, Lv ZY, Wang XY, Tian CH, Xu YP, Zhang CX (2009). Gene expression profiling of resistant and susceptible Bombyx mori strains reveals nucleopolyhedrovirus-associated variations in host gene transcript levels. Genomics 94 (2): 138-145.

Bettencourt R, Gunne H, Gastinel L, Steiner H, Faye I (1999). Implications of hemolin glycosylation and $\mathrm{Ca} 2+-$ binding on homophilic and cellular interactions. Eur. J. Biochem. 266 (3): 964-976.

Blasco R, Moss B (1991). Extracellular vaccinia virus formation and cellto-cell virus transmission are prevented by deletion of the gene encoding the 37,000-Dalton outer envelope protein. J. Virol. 65 (11): 5910-5920.

Bulet P, Hetru C, Dimarcq JL, Hoffmann D (1999). Antimicrobial peptides in insects; structure and function. Dev. Comp. Immunol. 23 (4-5), 329-344.

Chen KP, Lin CQ, Yao Q (1996). Studies on the resistance to NPV and its heredity regularity in the silkworm, Bombyx mori L. Acta Seri. Sinica 22: 160-164.

Chernysh S, Kim SI, Bekker G, Pleskach VA, Filatova NA, Anikin VB, Platonov VG, Bulet $P$ (2002). Antiviral and antitumor peptides from insects. PNAS 99 (20): 12628-12632.

Choudhari MK, Punekar SA, Ranade RV, Paknikar KM (2012). Antimicrobial activity of stingless bee (Trigona $s p$.) propolis used in the folk medicine of Western Maharashtra, India. J. Ethnopharmacol. 141 (1): 363-367.

Clem RJ, Fechheimer M, Miller LK (1991). Prevention of apoptosis by a baculovirus gene during infection of insect cells. Science 254 (5036): 1388-1390.

De Gregorio E, Han SJ, Lee WJ, Baek MJ, Osaki T, Kawabata S, Lee BL, Iwanaga S, Lemaitre B, Brey PT (2002). An immune-responsive Serpin regulates the melanization cascade in Drosophila. Dev. Cell 3 (4): 581-592.

Fuxa JR, Richter AR (1998). Repeated reversion of resistance to ucleopolyhedrovirus by anticarsia gemmatalis. J. Invertebr. Pathol. 71 (2): 159-164.

Haas-Stapleton EJ, Washburn JO, Volkman LE (2003). Pathogenesis of Autographa californica $\mathrm{M}$ nucleopolyhedrovirus in fifth instar Spodoptera frugiperda. J. Gen. Virol. 84: 2033-2040.

Hara S, Yamakawa M (1995a). Moricin, a novel type of antibacterial peptide isolated from the silkworm, Bombyx mori. J. Biol. Chem. 270 (50): 29923-29927.

Hara S, Yamakawa M (1995b). A novel antibacterial peptide family isolated from the silkworm, Bombyx mori. Biochem. J. 310: 651-656.

Hayama K (1978). Red fluorescent protein in the digestive juice of the silkworm larvae fed on host-plant mulberry leaves. Ent. Exp. Appl. 24: 228-236.

Hayama K, Nishida J (1976). The mechanism of formation of the red fluorescent protein in the digestive juice of silkworm larvae-the formation of chlorophyllidae-a. J. Appl. Entomol. Zool. 20: 37-43 (in Japanese).
Hirai M, Terenius O, Li W, Faye I (2004). Baculovirus and dsRNA induce Hemolin, but no antibacterial activity, in Antheraea pernyi. Insect Mol. Biol. 13 (4): 399-405.

Hoffmann JA (2003). The immune response of Drosophila. Nature 426 (6962): 33-38.

Hu XL, Chen L, Xiang XW, Yang R, Yu SF, Wu XF (2012). Proteomic analysis of peritrophic membrane (PM) from the midgut of fifth-instar larvae, Bombyx mori. Mol. Biol. Rep. 39 (4): 3427-3434.

Ito K, Kidokoro K., Sezutsu H, Nohata J, Yamamoto K, Kobayashi I, Uchino K, Kalyebi A, Eguchi R, Hara W, Tamura T, Katsuma S, Shimada T, Mita K, Kadono-Okuda K (2008). Deletion of a gene encoding an amino acid transporter in the midgut membrane causes resistance to a Bombyx parvo-like virus. PNAS 105 (21): 7523-7527.

Jakubowska AK, Lynn DE, Herrero S, Vlak JM, Oers MM (2009). Hostrange expansion of Spodoptera exigua multiple nucleopolyhedrovirus to Agrotis segetum larvae when the midgut is bypassed. J. Gen. Virol. 91 (4): 898-906.

Kamil JP, Tischer BK, Trapp S, Nair VK, Osterrieder N, Kung HJ (2005). VLIP, a viral lipase homologue, is a virulence factor of Marek's disease virus. J. Virol. 79 (11): 6984-6996.

Katsuma S, Mita K, Shimada T (2007). ERK- and JNK-dependent signaling pathways contribute to Bombyx mori nucleopolyhedrovirus infection. J. Virol. 81: 13700-13709.

Keddie BA, Aponte GW, Volkman LE (1989). The pathway of infection of Autographa californiaca nuclear polyhedrosis virus in an insect host. Science 243: 1728-1730.

Kenneth W, Sheena CC, Andrew FR, Judith KP (2001). Melanism and disease resistance in insects. Eco. Lett. 4: 637-649.

Kim DW, Hwang HS, Kim DS, Sheen SH, Heo DH, Hwang G, Kang SH, Kweon H, Jo YY, Kang SW, Lee KG, Park KW, Han KH, Park J, Eum WS, Cho YJ, Choi HC, Choi SY (2011). Effect of silk fibroin peptide derived from silkworm Bombyx mori on the anti-inflammatory effect of Tat-SOD in a mice edema model. BMB Rep. 44 (12): 787-792.

Kirkpatrick BA, Washburn JO, Volkman LE (1998). AcMNPV pathogenesis and developmental resistance in fifth instar Heliothis virescens. J. Invertebr. Pathol. 72 (1): 63-72.

Lavine MD, Strand MR (2002). Insect hemocytes and their role in immunity. Insect Biochem. Mol. Biol. 32 (10): 1295-1309.

Lehane MJ (1997). Peritrophic matrix structure and function. Annu. Rev. Entomol. 42: 525-550.

Levashina EA, Moita LF, Blandin S, Vriend G, Lagueux M, Kafatos FC (2001). Conserved role of a complement-like protein in phagocytosis revealed by dsRNA knockout in cultured cells of the mosquito, Anopheles gambiae. Cell 104 (5): 709-718.

Ligoxygakis P, Pelte N, Ji C, Leclerc V, Duvic B, Belvin M, Jiang H, Hoffmann JA, Reichhart JM (2002). A serpin mutant links Toll activation to melanization in the host defence of Drosophila. EMBO J. 21 (23): 6330-6337.

Liu X, Yao Q, Wang Y, Chen KP (2010). Proteomic analysis of nucleopolyhedrovirus infection resistance in the silkworm, Bombyx mori (Lepidoptera: Bombycidae). J. Invertebr. Pathol. 105 (1): 84-90.

Meister M, Lagueux M (2003). Drosophila blood cells. Cell Microbiol. 5 (9): 573-580.

Monsma SA, Oomens AG, Blissard GW (1996). The GP64 envelope fusion protein is an essential baculovirus protein required for cell-tocell transmission of infection. J. Virol. 70 (7): 4607-4616.

Moreno-Habel DA, Biglang-awa IM, Dulce A, Luu DD, Garcia P, Weers PM, Haas-Stapleton EJ (2012). Inactivation of the budded virus of Autographa californica $\mathrm{M}$ nucleopolyhedrovirus by gloverin. J. Invertebr. Pathol. 110 (1): 92-101.

Moscardi $F$ (1999). Assessment of the application of baculoviruses for control of Lepidoptera. Annu. Rev. Entomol. 44: 257-289.

Nakazawa H, Tsuneishi E, Ponnuvel KM, Furukawa S, Asaoka A, Tanaka H, Ishibashi J, Yamakawa M (2004). Antiviral activity of a serine protease from the digestive juice of Bombyx mori larvae against nucleopolyhedrovirus. Virology 321 (1): 154-162.

Ourth DD (2004). Antiviral activity against human immunodeficiency virus-1 in vitro by myristoylated-peptide from Heliothis virescens. Biochem. Biophys. Res. Comm. 320 (1): 190-196.

Pandian GN, Ishikawa T, Togashi M, Shitomi Y, Haginoya K, Yamamoto S, Nishiumi T, Hori H (2008). Bombyx mori midgut membrane protein $\mathrm{P} 252$, which binds to Bacillus thuringiensis Cry1 A, is a chlorophyllide- 
binding protein, and the resulting complex has antimicrobial activity. Appl. Environ. Microbiol. 74 (5): 1324-1331.

Ponnuvel KM, Nakazawa H, Furukawa S, Asaoka A, Ishibashi J, Tanaka $\mathrm{H}$, Yamakawa M (2003). A lipase isolated from the silkworm Bombyx mori shows antiviral activity against nucleopolyhedrovirus. J. Virol. 77 (19): 10725-10729.

Popham HJ, Shelby KS, Brandt SL, Coudron TA (2004). Potent virucidal activity in larval Heliothis virescens plasma against Helicoverpa zea single capsid nucleopolyhedrovirus. J. Gen. Virol. 85 (8): 2255-2261.

Prudhomme JC, Couble P (2002). Perspectives in silkworm transgenesis. Curr. Sci. 83 (4): $432-438$.

Qin LG, Xia HC, Shi HF, Zhou YJ, Chen L, Yao Q, Liu XY, Feng F, Yuan Y, Chen KP (2012). Comparative proteomic analysis reveals that caspase-1 and serine protease may be involved in silkworm resistance to Bombyx mori nuclear polyhedrosis virus. J. Proteomics 75 (12): 3630-3638.

Rahman MM, Gopinathan KP (2004). Systemic and in vitro infection process of Bombyx mori nucleopolyhedrovirus. Virus Res. 101: 109118.

Selot R, Kumar V, Shukla S, Chandrakuntal K, Brahmaraju M, Dandin SB, Laloraya M, Kumar PG (2007). Identification of a soluble NADPH oxidoreductase (BmNOX) with antiviral activities in the gut juice of Bombyx mori. Biosci. Biotechnol. Biochem. 71 (1): 200-205.

Smede M, Hussain M, Asgari S (2009). A lipase-like gene from Heliothis virescens ascovirus (HvAV-3e) is essential for virus replication and cell cleavage. Virus Genes 39 (3): 409-417.

Sunagar SG, Lakkappan VJ, Ingalhalli SS, Savanurmath CJ, Hinchigeri SB (2008). Characterization of the photochromic pigments in red fluorescent proteins purified from the gut juice of the silkworm Bombyx mori L. Photochem. Photobiol. 84 (6): 1440-1444.

Sunagar SG, Savanurmath CJ, Hinchigeri SB (2011). The profiles of red fluorescent proteins with antinucleopolyhedrovirus activity in races of the silkworm Bombyx mori. J. Insect Physiol. 57 (12): 1707-1714.
Tamura T, Thibert C, Royer C, Kanda T, Abraham E, Kamba M, Komoto N, Thomas JL, Mauchamp B, Chavancy G, Shirk P, Fraser M,

Prudhomme JC, Couble P (2000). Germline transformation of the silkworm Bombyx mori L. using a piggyBac transposon-derived vector. Nat. Biotechnol. 18: 81-84.

Teakle RE, Jensen JM, Giles JE (1986). Age-related susceptibility of Heliothis punctiger to a commercial formulation of nuclear polyhedrosis virus. J. invertebr. Pathol. 47: 82-92.

Trudeau D, Washburn JO, Volkman LE (2001). Central role of hemocytes in Autographa californica M nucleopolyhedrovirus pathogenesis in Heliothis virescens and Helicoverpa zea. J. Virol. 75 (2): 996-1003.

Tzou P, Ohresser S, Ferrandon D, Capovilla M, Reichhart JM, Lemaitre B, Hoffmann JA, Imler JL (2000). Tissue-specific inducible expression of antimicrobial peptide genes in Drosophila surface epithelia. Immunity 13 (5): 737-748.

Vernick KD, Fujioka H, Seeley DC, Tandler B, Aikawa M, Miller LH (1995). Plasmodium gallinaceum: a refractory mechanism of ookinete killing in the mosquito, Anopheles gambiae. Exp. Parasitol. 80 (4): 583-595.

Washburn JO, Kirkpatrick BA, Volkman LE (1995). Comparative pathogenesis of Autographa californica M nuclear polyhedrosis virus in larvae of Trichoplusia ni and Heliothis virescens. Virology 209 (2): 561-568.

Xiang ZH (1995). Genetics and Breeding of the Silkworm. Chinese Agriculture Press, Beijing, pp. 273-289.

Zhao P, Dong ZM, Duan J, Wang GH, Wang, LY, Li YS, Xiang ZH, Xia QY (2012). Genome-wide identification and immune response analysis of serine protease inhibitor genes in the silkworm, Bombyx mori. PLoS One 7 (2): e31168. 